\title{
Cooperative Adaptive Threshold Based Energy and Matched Filter Detector in Cognitive Radio Networks
}

\author{
Ashish Rauniyar, ${ }^{1,2}$ and Soo Young Shin ${ }^{1}$ \\ 1. Wireless Emerging Networking System Lab, Kumoh National Institute of Technology, 730-701, South-Korea \\ 2. College of Computer \& Information Engineering, Hohai University, 210092, China
}

\begin{abstract}
CR (cognitive radio) is the key technology for unlicensed SUs (secondary users) to exploit the unused spectrum of PUs (primary users) via opportunistic spectrum access. In this paper, we propose a new spectrum sensing method using cooperative adaptive threshold based on energy and matched filter detector in cognitive radio networks. The ED (energy detector) is highly susceptible to noise uncertainty condition. Also, ED cannot well differentiate between the signal and noise, if the detected observational value of the PU lies in the confused region i.e., between signal and noise. We propose a scheme based on cooperative adaptive threshold that uses MF (matched filter) detector as a second stage detector for the reliable detection in the confused region and energy efficient ED as first stage detector for the clear region where the detector can easily differentiate between signal and noise and makes its own local decision. The fusion center collects the local decisions and observational values of the secondary users and then makes the final decision to ascertain whether the primary user is present or not. Simulation results shows that our proposed method has higher detection performance as compared to other spectrum sensing methods.
\end{abstract}

Key words: Cognitive radio, energy detector, matched filter, adaptive threshold.

\section{Introduction}

The rapid growth in wireless communications has contributed to a huge demand on the deployment of new wireless services in both the licensed and unlicensed frequency spectrum. However, in a survey conducted by the FCC (federal communications commission) on spectrum utilization has indicated that the actual licensed spectrum is largely under-utilized in vast geographical dimensions [1]. CR provides opportunistic access to unused licensed bands [2, 3]. In CR systems, the unlicensed users can utilize the licensed frequencies while the PU is not active. In the recent years, CSS (cooperative spectrum sensing) scheme has become a popular technique to solve the inefficiency of spectrum usage and provide high level of protection to the PU from SU. In CR, sensing accuracy is important for avoiding interference to the primary users. Reliable spectrum sensing is not

Corresponding author: Soo Young Shin, professor, research fields: cognitive radio, WLAN, WiMAX. E-mail: wdragon@kumoh.ac.kr. always guaranteed due to multipath fading, shadowing and hidden terminal problem. Cooperative spectrum sensing has thus been introduced for quick and reliable detection [4-7]. The CSS has two successive stages, sensing and reporting. In sensing stage, spectrum sensing is done by several local SU. Then in next stage, PU sensing decisions or measurements is sent to FC (fusion center) to combine them and make a better overall decision.

Among several spectrum sensing techniques, ED (energy detector) is the most popular method employed for spectrum sensing. Measuring only the received signal power and comparing it with a pre-fixed threshold, the ED is a non-coherent detection device with low implementation complexity and is more power efficient. But ED performance is highly degraded under noise uncertainty condition [8]. Also, ED cannot well differentiate between the signal and noise, if the detected observational values lies in the confused region i.e., between signal and noise.

In Ref. [9], a censoring method using double 
threshold based on ED was proposed. If the detected observational energy values $\left(O_{i}\right)$ by the SU lies in the confused region, they will not report to the fusion center. This method can reduce the sensing time and cause sensing failure problem. Paper [10] also proposed a method using double threshold based on ED to increase the detection performance as compared to the conventional ED. In this method, first SU will make the local decision by comparing their $O_{i}$ of the clear region with the pre-defined threshold of ED. If the $O_{i}$ lies in the confused region, then the $\mathrm{SU}$ will forward it to the FC. The FC will make overall decision by considering the local decision of $\mathrm{SU}$ of clear region and comparing the $O_{i}$ of confused region with another threshold value of ED.

To overcome the noise uncertainty problem of ED and to increase the detection performance, we propose a new CSS technique using cooperative adaptive threshold based on ED and MF (matched filter) detector. Our proposed scheme has higher detection performance as compared to other conventional methods and the method described in Ref. [10]. We take the advantages of energy efficient ED to make the local decision in the clear region and reliable MF to take the decision in the confused region. We further divide the confused region into equal quantization intervals to take the decision accordingly. Simulation results show that our proposed scheme has higher detection performance, lower miss-detection probability and it can perform well in low SNR as compared to other methods.

The rest of the paper is organized as follows: Section 2 presents the system description; Section 3 describes our proposed model. Simulation results are shown in Section 4; Finally conclusion is drawn in Section 5.

\section{System Description}

The main aim of $\mathrm{CR}$ is to correctly identify the presence of PU and allows the SUs to utilize the unused spectrum, if it is not used by licensed PUs. Under binary hypothesis testing, we consider the occurrence of two input events in observing signal $x_{i}$ in some observation interval denoted by

$$
x_{i}=\left\{\begin{array}{cc}
n_{i} & H_{0} \\
s_{i}+n_{i} & H_{1}
\end{array}\right.
$$

where, $i=1,2,3, \ldots N$ is number of samples. $H_{0}$ represents the hypothesis that the observation vector consists of noise. $H_{1}$ represents the hypothesis that the observation vector consists of noise and signal. The noise component $n_{i}$ is assumed to be additive white gaussian random variable, which is independent and identically-distributed (i. i. d) with zero mean normal distribution with variance $\sigma^{2} \sim N\left(0, \sigma^{2}\right)$, and $s_{i}$ is the signal.

\subsection{Energy Detector}

The ED is non-coherent detector and consumes less amount power. ED detects the presence of signals by simply squaring its energy and comparing that energy around the carrier frequency with certain threshold [11]. The ED is not accurate as the detected signal can be affected by noise level. The performance of ED is highly degraded under noise uncertainty condition. Fig. 1 shows the energy distribution of the primary user signal and noise.

The ED consists of a quadrature receiver with $y_{I}$ and $y_{Q}$ representing samples from In-phase and quadrature branch respectively. The samples after passing the

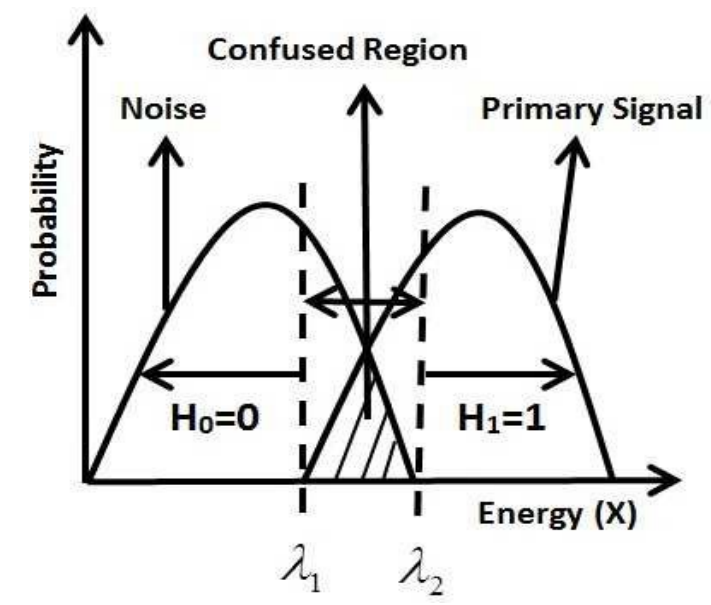

Fig. 1 Energy distribution of primary user signal and noise. 
squaring device, output of the integrator is denoted by

$$
y_{I}=y_{Q}=\left(\frac{1}{N_{0}}\right) \int_{0}^{T} r^{2}(t) d t
$$

where, $r(\mathrm{t})$ is input signal, $N_{0}$ is noise spectral density.

Within observed sensing period, test statistic ED can be approximated as $Y_{E D}=y_{I}+y_{Q}$. At the observation time $t$, decision variable $Y_{E D}$ will be compared to a detection threshold of ED denoted by $\lambda^{E D}$. Threshold value is set to meet the target probability of false alarm $p_{f}$ according to the noise power. The probability of detection $p_{d}$ can be also identified. The expression for $p_{f}$ and $p_{d}$ can be given as: [12]

$$
p_{f a}^{E D}=1-F_{x}\left(\frac{\lambda^{E D}}{\sigma^{2}}, \quad 2 n\right)
$$

where, $F_{\chi}$ is $\mathrm{CDF}$ (cumulative distribution function) of standard chi-square random variable with $k$ degree of freedom.

$$
p_{d}^{E D}=Q\left(\sqrt{n(S N R)}, \quad \sqrt{\frac{\lambda^{E D}}{\sigma^{2}}}\right)
$$

where, $Q$ is generalized Marcum-Q function.

\subsection{Matched Filter}

MF is a reliable detector but consumes high amount of power. MF works using receivers bank of $L$ matched filters, which runs together to correlate the incoming signals [13]. At each sampling instant $t$, de-correlator process signal $x(t)$, the output on interval

$(0, T)$ that contains two sample output from a module is given by

$$
Y_{M F}={y_{I_{i}}}^{2}+y_{Q_{i}}{ }^{2}, i=1,2, \ldots \ldots, L
$$

The $Y_{M F}$ forms $L$ decorrelator output in which we find the decision variable $V$ from the maximum of $Y_{M F}$ over $M$ offset bits. Variable $V$ is compared to threshold $\lambda^{M F}$ to decide the presence or absence of signal.

$$
V=\max \left\{Y_{M F}^{m}\right\}, m=1, \ldots . M
$$

The acquisition process of MF will give probability of false alarm and probability of detection that can be calculated as: [12]

$$
\begin{gathered}
p_{f a}^{M F}=1-F_{x}\left(\frac{\lambda^{M F}}{\sigma}, 2\right) \\
p_{d}^{M F}=Q\left(\sqrt{2 n(S N R)}, \sqrt{\frac{\lambda^{M F}}{\sigma^{2}}}\right)
\end{gathered}
$$

where, $\lambda^{M F}$ is the threshold setting for MF, the non-centrality parameter $s^{2}=2 n(S N R)$ is the output of the filters in $I$ and $Q$ branches at the correct offset. The correlation process of MF has a central chi-square distribution with $\sqrt{2}$ degree of freedom with variance $(\sigma=\sqrt{n})$.

\section{Cooperative Adaptive Threshold Based Energy and Matched Filter Detector}

In conventional ED, each $\mathrm{SU}$ makes their own local decision whether PU is present $\left(H_{1}\right)$ or PU is absent $\left(H_{0}\right)$ by comparing the $O_{i}$ with single predefined threshold. The main idea of our proposed scheme is that we take the advantage of energy efficient ED to make decision in the clear region as a first stage detector and reliable MF to make decision in the confused region as second stage detector. Our proposed scheme is based on cooperative adaptive threshold of both ED and MF detector.

Fig. 2 shows the working model of our proposed method. Each SU of the CSS are equipped with ED and performs spectrum sensing individually. The observational value $O_{i}$ of $\mathrm{SU}$ is checked with the threshold values $\lambda_{1}^{E D}$ and $\lambda_{2}^{E D}$ of $E D$ and the decision will be taken accordingly. If $O_{i}$ satisfies $\lambda_{1}^{E D} \leq O_{i} \leq \lambda_{2}^{E D}$, which means that the PU signal lies

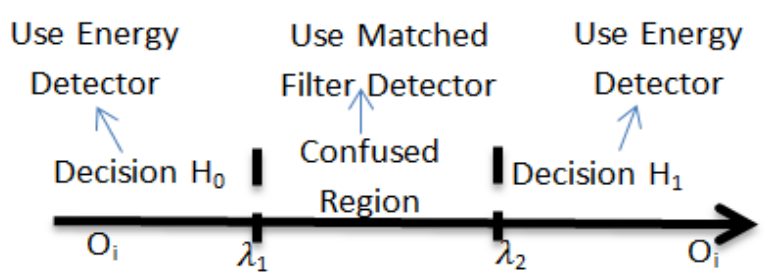

Fig. 2 Proposed detection method. 


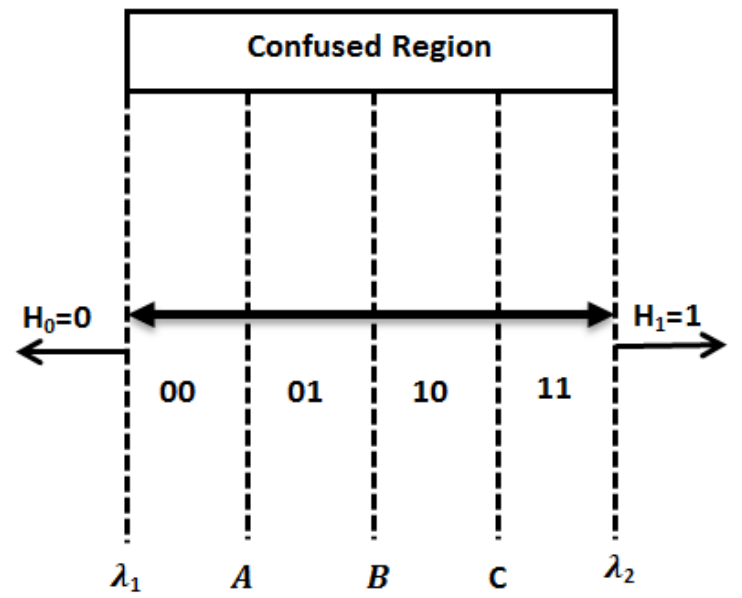

Fig. 3 Division of confused region into four equal quantization intervals using two-bit quantization method.

in the confused region, then Decision D will be taken based on their quantization interval of the confused region. The local decision $L_{i}$ is given by

$$
L_{i}=\left\{\begin{array}{ccrl}
0 & & 0 & \leq O_{i} \leq \lambda_{1}^{E D} \\
D=H & \lambda_{1}^{E D} & \leq O_{i} \leq \lambda_{2}^{E D} \\
1 & O_{i} & \geq \lambda_{2}^{E D}
\end{array}\right.
$$

Fig. 3 shows the two-bit quantization method which divides confused region into four equal quantization intervals as $\left(\lambda_{1} A-A B-B C-C \lambda_{2}\right), S$ is the equal gap between each quantization levels. $\lambda_{1}, A, B, C$, and $\lambda_{2}$ are sub-thresholds (ST), and their values are chosen as

$$
S T=\left\{\begin{array}{l}
A=\lambda_{1}+S \\
B=A+S \\
C=B+S \\
\lambda_{2}=C+S
\end{array}\right.
$$

where, $S=\frac{\lambda_{2}-\lambda_{1}}{4}$ and decision $D$ is given by

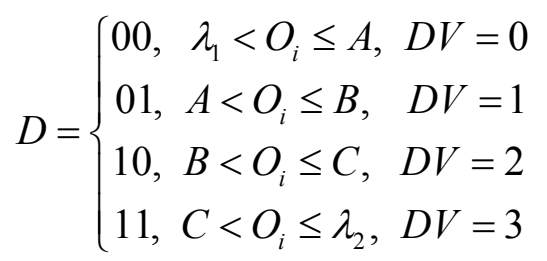

If $O_{i}$ fall inside any of the quantized intervals of the confused region, then its respective DV (decimal values) will be generated as shown by Eq. (11).

Without the loss of generality, we assume that fusion center FC receives $K$ local decisions of clear region out of $N$ SUs. Then $N-K$ observational values $O_{i}$ of confused region will be reported to the $\mathrm{FC}$ to make the quantization decision based on their DV. Eq. (11), gives two-bits binary values of the respective quantized levels. Then, the DV checks the values of D and gives its respective decimal values accordingly. Further, the fusion center will now apply more reliable $\mathrm{MF}$ as a second stage detector on those $N-K O_{i}$ based on their quantization level and respective DV generated. The threshold of MF $\lambda^{M F}$ at the FC is chosen according to the appropriate false alarm probability of MF as given by Eq. (7). The decision $D^{F}$ at the FC using the MF detector on $N-K O_{i}$ is as follows:

$$
D^{F}=\left\{\begin{array}{cc}
0 & 0 \leq \sum_{i=1}^{N-K} O_{i} \leq \lambda^{M F} \\
1 & \sum_{i=1}^{N-K} O_{i}>\lambda^{M F}
\end{array}\right.
$$

The FC has the local decision $L_{i}$ of $K$ SUs using ED and decision $D$ of $N-K$ SUs using MF. Let us denote the total decision at FC by $Z$, i.e., $Z=D^{F}+\sum_{i=1}^{K} L_{i}$. The FC makes a final decision using a hard decision OR rule for deciding the presence or absence of PU. As per the hard decision OR rule, if total decision $\mathrm{Z}$ is greater or equal to 1 then signal is detected $\left(H_{l}\right)$ and if $Z$ is smaller than 1 then signal is not detected $\left(H_{0}\right)$. The mathematical expression of hypothesis at the FC can be written as:

$$
F C= \begin{cases}Z<1, & H_{0} \\ Z \geq 1, & H_{1}\end{cases}
$$

(1) Cooperative Detection and False Alarm Probabilities of Proposed Method: First each secondary user decides either ' 0 ' or ' 1 ' or 'No Decision" on the basis of comparison of $O_{i}$ with pre-defined threshold value of energy detector. Decision goes in favor of ' 0 ' if PU is absent. Similarly decision goes in favor of ' 1 ', if the PU is present. Let us denote the probability of deciding ' 1 ', probability of "No Decision" and probability of deciding ' 0 ' 
under hypothesis $H_{1}$ is represented by, $p_{d 1}^{E D}, \Delta_{1, i}^{E D}$ and $p_{m}^{E D} \quad$ respectively. Similarly, Probability of deciding '1', probability of "No Decision" and probability of deciding ' 0 ' under hypothesis $H_{0}$ is denoted by $p_{f a 0}^{E D}, \Delta_{1, i}^{E D}$ and $p_{d 0}^{E D}$ respectively. The expressions for different probabilities are given below considering the AWGN channel $[9,14]$.

$$
\begin{gathered}
p_{d 1}^{E D}=P\left\{O_{i}>\lambda_{2}^{E D} \mid \mathrm{H}_{1}\right\}=Q\left(\sqrt{n(S N R)}, \sqrt{\lambda_{2}^{E D}}\right) \\
p_{d 0}^{E D}=P\left\{O_{i}<\lambda_{1}^{E D} \mid \mathrm{H}_{0}\right\}=\mathrm{F}_{x}\left(\lambda_{1}^{E D}, 2\right) \\
\Delta_{1, i}^{E D}=P\left\{\lambda_{1}^{E D}<O_{i}<\lambda_{2}^{E D} \mid H_{1}\right\} \\
\Delta_{0, i}^{E D}=P\left\{\lambda_{1}^{E D}<O_{i}<\lambda_{2}^{E D} \mid H_{0}\right\} \\
p_{m}^{E D}=P\left\{O_{i} \leq \lambda_{1}^{E D} \mid H_{1}\right\}=1-\Delta_{1, i}^{E D}-p_{d 1}^{E D} \\
p_{f a 0}^{E D}=P\left\{O_{i}>\lambda_{2}^{E D} \mid H_{0}\right\}=1-F_{x}\left(\lambda_{2}^{E D}, 2\right)
\end{gathered}
$$

The cooperative probability of detection $Q_{d}$ of the FC using $O R$ rule as indicated in Eq. (11) can be expressed as:

$$
\begin{aligned}
& Q_{d}=1-\sum_{K=0}^{N-1}\left(\begin{array}{l}
N \\
K
\end{array}\right) \prod_{i=1}^{K} p_{m}^{E D} . \\
& \prod_{i=K+1}^{N} \Delta_{1, i}^{E D}\left[1-Q_{(N-K) u}\left(\sqrt{2 n(S N R)} \quad \sqrt{\lambda^{M F}}\right)\right](20) \\
& +\prod_{i=1}^{N} p_{m}^{E D}
\end{aligned}
$$

where, $u$ is the time bandwidth product. The cooperative probability of miss-detection $Q_{m}$ of the $\mathrm{FC}$ is given by

$$
Q_{m}=1-Q_{d}
$$

The cooperative probability of false alarm $Q_{f}$ of the FC using $O R$ rule as indicated in Eq. (11) can be expressed as:

$$
\begin{aligned}
& Q_{f}=-\sum_{K=0}^{N-1}\left(\begin{array}{l}
N \\
K
\end{array}\right) \prod_{i=1}^{K}\left(1-\Delta_{0, i}^{E D}-p_{f a 0}^{E D}\right) . \\
& \prod_{i=K+1}^{N} \Delta_{0, i}^{E D}\left[1-F_{x(N-K) u}\left(\lambda^{M F}, 2\right)\right]
\end{aligned}
$$

\section{Simulation Results}

Our simulation was conducted in MATLAB to investigate the performance of our proposed scheme. AWGN is imposed on the original signal xi either for $H_{0}$ or $H_{1}$ condition. We assume that there is error free control channel available between the secondary users and the fusion center at the base station for sending local decisions and observational values $O_{i}$ of the confused region.

The ROC (receiver operating characteristics) curves of our proposed scheme as compared to other schemes is shown in Fig. 4. The ROC curve is obtained with $S N R=10 \mathrm{~dB}$, Number of cooperative $S U_{S}=10$, $\Delta_{0, i}^{E D}=\Delta_{1, i}^{E D}=0.1$, time bandwidth product $u=5$.

Clearly our proposed scheme has the higher detection performance compared to other double threshold method using ED only and conventional ED. Our scheme takes the advantage of reliable MF detector in the confused region to take the decision.

Fig. 5 shows cooperative miss-detection probability curve of our proposed scheme as compared to other schemes. With the use of MF, our scheme is able to differentiate the signal and noise in the confused region and it can take decision accordingly. As expected our proposed scheme miss-detection probability is lower as compared to the previous schemes explained in the literature.

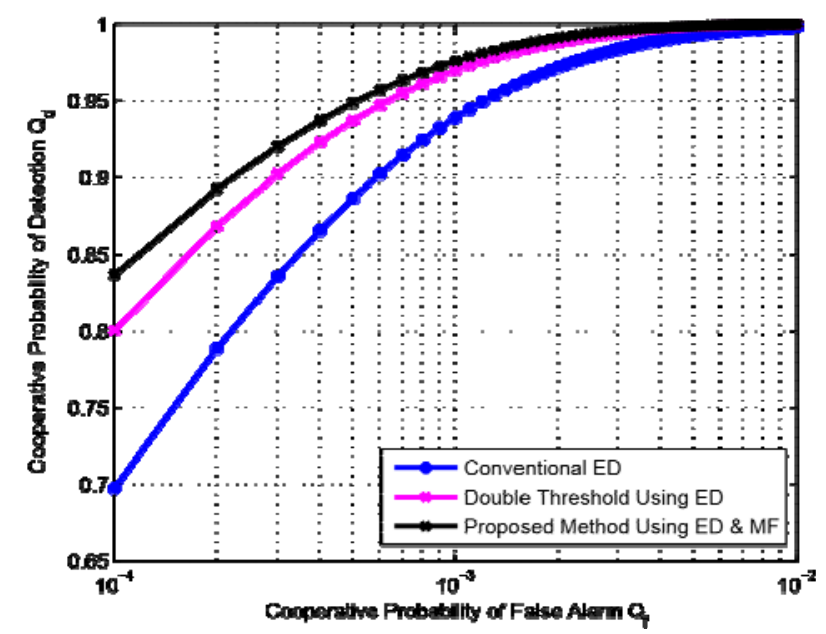

Fig. 4 ROC of our proposed scheme using cooperative adaptive threshold based energy and matched filter detector. 


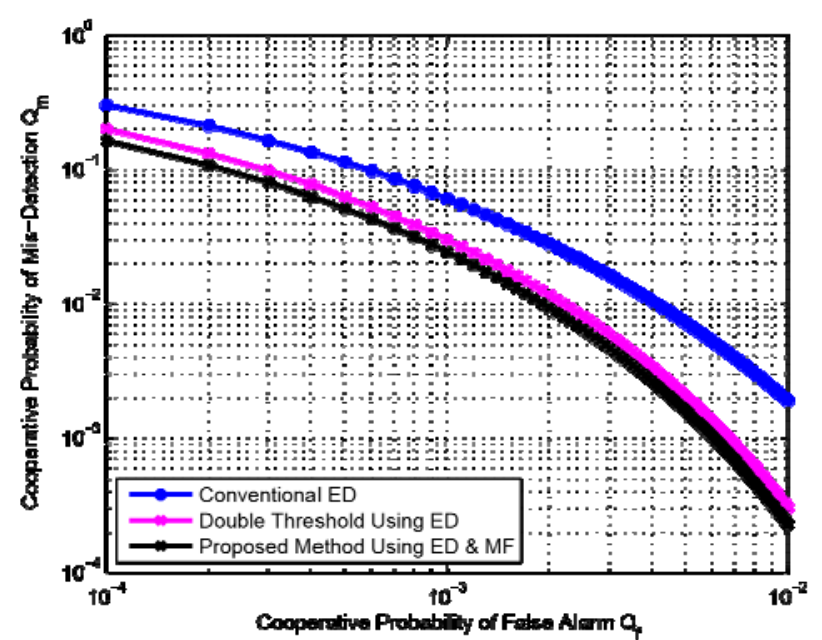

Fig. 5 Comparison of cooperative miss detection probability of our proposed scheme with other.

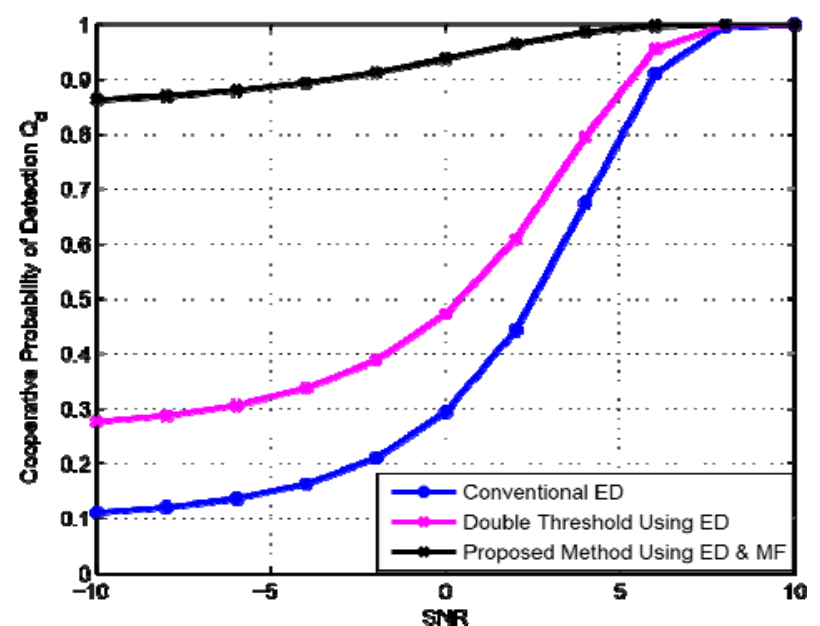

Fig. 6 Comparison of cooperative probability of detection of our proposed scheme with other methods at different SNR.

Fig. 6 shows the cooperative probability of detection curves against different SNR values. Fig. 6 is plotted using the probability of false alarm of energy detector is set at 0.01 i.e., $p_{f a}^{E D}=0.01, S N R$ values ranges from $-10 d B$ to $10 d B$, number of cooperative $S U s=10, \Delta_{0, i}^{E D}=\Delta_{1, i}^{E D}=0.01$, time bandwidth product $u=5$. It is clear from Fig. 6 that our proposed scheme outperforms the other schemes at different SNR ranges. Even at $-10 \mathrm{~dB}$ SNR value, our scheme is clearly able to detect the signal as compared to other schemes. The scheme only using double threshold conventional energy detector suffer greatly at low SNR region is due to the fact that the energy detector is highly susceptible to the noise uncertainty at the low SNR. The decision in the confused region is clearly indicated by the quantization decision of MF in our scheme. Hence, our proposed scheme performance is superior to all other scheme.

\section{Conclusion}

In this paper, we have proposed a new adaptive double-threshold based energy and matched filter detector for cognitive radio networks. The proposed method gives significantly better detection performance compared to other methods. Also, the cooperative probability of miss-detection of our proposed scheme is lower than other scheme. At lower SNR region, energy detector cannot differentiate between signal and noise and is susceptible to noise uncertainty. Our proposed scheme takes the advantage of energy efficient energy detector to take decision in the clear region and reliable matched filter detector to take decision in the confused region. Hence our proposed scheme performance is better compared to other schemes.

\section{Acknowledgment}

This research was supported by Basic Science Research Program through the NRF (national research foundation) of Korea funded by the Ministry of Science, ICT and Future Planning (2012R1A1A1009442).

\section{References}

[1] Akyildiz, I. F. 2002. Federal Communications Commission et al.: Spectrum Policy Task Force. Rep. et Docket.

[2] Mitola, J., and Maguire, G. Q. J. 1999. "Cognitive Radio: Making Software Radios More Personal.” IEEE Personal Communications 6 (Aug.): 13-8.

[3] Haykin, S. 2005. "Cognitive Radio: Brain-Empowered Wireless Communications." IEEE Journal on Selected Areas in Communications 23 (February): 201-20.

[4] Ganesan, G., and Li, Y. 2007. "Cooperative Spectrum Sensing in Cognitive Radio, part ii: Multiuser networks." IEEE Transactions on Wireless Communications 6 (June): 
2214-22.

[5] Fan, R. F., and Jiang, H. 2010. "Optimal Multi-Channel Cooperative Sensing in Cognitive Radio Networks." IEEE Transactions on Wireless Communications 9 (March): 1128-38.

[6] Zhang, W., Mallik, R. K., and Letaief, K. 2009. "Optimization of Cooperative Spectrum Sensing with Energy Detection in Cognitive Radio Networks." IEEE Transactions on Wireless Communications 8 (December): 5761-66.

[7] Mishra, S. M., Sahai, A., and Brodersen, R. W. 2006. "Cooperative Sensing Among Cognitive Radios." Presented at the IEEE International Conference on Communications.

[8] Park, K. Y. 1978. "Performance Evaluation of Energy Detectors." IEEE Transactions on Aerospace and Electronic Systems 14 (March): 237-41.

[9] Sun, C., Zhang, W., and Letaief, K. 2007. "Cooperative Spectrum Sensing for Cognitive Radios under Bandwidth Constraints." Presented at the Wireless Communications and Networking Conference.

[10] Zhu, J., Xu, Z. G., Wang, F. R., Huang, B. X., and Zhang, B. 2008. "Double Threshold Energy Detection of
Cooperative Spectrum Sensing in Cognitive Radio." Presented at the 3rd International Conference on Cognitive Radio Oriented Wireless Networks and Communications.

[11] Lehtomaki, J. J., Vartiainen, J., Htoniemi, R. V., and Saarnisaari, H. 2011. "Adaptive Fcme-Based Threshold Setting for Energy Detectors." In Proceedings of the 4th International Conference on Cognitive Radio and Advanced Spectrum Management.

[12] Triwicaksono, D., and Shin, S. Y. 2013. "Energy Detector and Matched Filter as Cascaded Clear Channel Assessment in Wireless Network." Presented at the IET International Conference on Information and Communications Technologies (IETICT 2013).

[13] Giannakis, G. B., and Tsatsanis, M. K. 1990. "Signal Detection and Classification Using Matched Filtering and Higher Order Statistics." IEEE Transactions on Acoustics, Speech and Signal Processing 38: 1284-96.

[14] Ashish, R., and Shin, S. Y. 2014. "Adaptive Double-Threshold Based Energy and Matched Filter Detector in Cognitive Radio Networks." In proceedings of International Workshop on Mobile and Sensor Networks. 